\title{
Fundamental Differences in Mechanical Behavior between Two Types of Crystals at the Nanoscale
}

\author{
Steffen Brinckmann, Ju-Young Kim, and Julia R. Greer \\ Division of Engineering and Applied Science, California Institute of Technology, 1200 E California Boulevard, \\ Pasadena, California 91125, USA
}

(Received 24 January 2008; published 17 April 2008)

\begin{abstract}
We present differences in the mechanical behavior of nanoscale gold and molybdenum single crystals. A significant strength increase is observed as the size is reduced to $100 \mathrm{~nm}$. Both nanocrystals exhibit discrete strain bursts during plastic deformation. We postulate that they arise from significant differences in the dislocation behavior. Dislocation starvation is the predominant mechanism of plasticity in nanoscale fcc crystals, while junction formation and hardening characterize bcc plasticity. A statistical analysis of strain bursts is performed as a function of size and compared with stochastic models.
\end{abstract}

DOI: 10.1103/PhysRevLett.100.155502

PACS numbers: 62.25.- $\mathrm{g}, 61.46 . \mathrm{Hk}, 81.07 .-\mathrm{b}, 81.16 . \mathrm{Rf}$

The mechanical behavior of crystals is dictated by dislocation motion in response to applied force. While it is difficult to observe the motion of individual dislocations, several correlations can be made between the microscopic stress-strain behavior and dislocation activity. In bulk, plasticity in metals occurs by the motion of dislocations, which multiply in the course of plastic deformation causing strain hardening. Although this fundamental concept is often assumed to be applicable to crystals of any dimensions, numerous recent studies have shown that conventional plasticity breaks down at the submicron scale. Recently, many experimental and computational investigations of fcc crystals ( $\mathrm{Au}, \mathrm{Al}, \mathrm{Cu}, \mathrm{Ni}$ ) have demonstrated a pronounced size effect, whose main premise is "smaller is stronger" [1-11]. In this work we investigate flow stress as a function of diameter in gold (fcc) and molybdenum (bcc) single crystal nanopillars subjected to uniaxial microcompression. The results that follow suggest that fcc and bcc crystals have fundamentally different plasticity mechanisms when reduced to nanoscale with significant strain hardening present in the latter and virtually none in the former. In a striking deviation from classical mechanics, there is a significant increase in strength as crystal size is reduced to $100 \mathrm{~nm}$; however, in gold crystals (fcc) the highest strength achieved represents $44 \%$ of its theoretical strength, while in molybdenum crystals (bcc) it is only $7 \%$. This suggests that plasticity in Au is likely controlled by nucleation of new dislocations rather than by interactions of the preexisting ones. On the contrary, the smallest molybdenum nanopillar achieves only $\sim 7 \%$ of its theoretical strength, implying that plasticity is likely driven by the intricate motion and interactions of dislocations inside the pillar rather than by nucleation events. These remarkable differences in mechanical response of fcc and bcc crystals to uniaxial microcompression challenge the applicability of conventional strain hardening to nanoscale crystals. Single crystal nanopillars described in this work were fabricated via the focused ion beam system and

subsequently uniaxially compressed along the $\langle 001\rangle$ direction in the nanoindenter with a flat punch tip of $30 \mu \mathrm{m}$ diameter. The specifics of fabrication and testing conditions are based on the authors' previous work [5]. Scanning electron microscope images of gold and molybdenum nanopillars are shown in Figs. 1(a) and 1(b). A representative image of a compressed gold nanopillar demonstrating symmetrical deformation is shown in Fig. 1(c), and a compressed Mo pillar is shown in Fig. 1(d).

The deformed images clearly show that multiple slip systems were activated and that compression of the pillar midsection (excluding end effects) was relatively homoge-

(a)

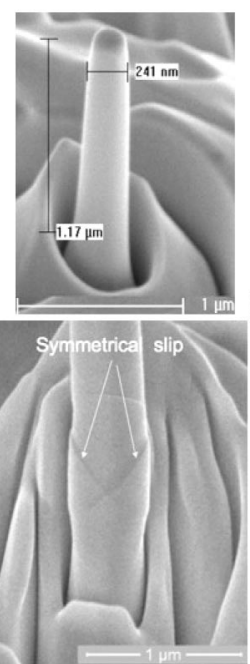

(c)

FIG. 1. (a) $\mathrm{Au}\langle 001\rangle$-oriented pillar before compression with initial diameter of $241 \mathrm{~nm}$. (b) Mo $\langle 001\rangle$-oriented pillar with initial diameter of $205 \mathrm{~nm}$. (c) Midsection of a representative gold pillar after compression showing symmetric double slip. (d) Same Mo pillar after compression. All images are shown at a $52^{\circ}$ tilt angle. Scanning electron microscope parameters: HV, high voltage; TLD, through the lens detector; WD, working distance; Acc. V, accelerating voltage. 
neous and symmetrical. Figure 2 shows the axial stressstrain curves generated based on the load-displacement data collected by the nanoindenter. Some of the nanopillars were compressed without intentional unloadings while others were unloaded and reloaded several times before reaching their final strain to ensure complete contact between the pillar and the indenter. All compression experiments were run in nominal constant displacement rate regime of $5 \mathrm{~nm} / \mathrm{s}$, corresponding to the strain rates between $3.33 \times 10^{-3} \mathrm{~s}^{-1}$ and $8.33 \times 10^{-3} \mathrm{~s}^{-1}$. The stress vs strain curves for several gold nanopillars with diameters ranging between $250 \mathrm{~nm}$ and $946 \mathrm{~nm}$ are shown in Fig. 2(a) and clearly reveal the presence of the size effect; i.e., smaller pillars require the application of higher stresses.

An interesting feature of these curves is that unlike in a typical smooth stress-strain plot of bulk single crystals, the stress-strain relationship for smaller pillars does not undergo stage II hardening but instead is composed exclusively of elastic loading segments followed by discrete displacement bursts. These bursts are likely correlated with the initiation of dislocation avalanches, activated in response to the shear stress in the glide plane. Contrary to the very discontinuous stress-strain behavior in gold, the results for molybdenum nanopillars subjected to the same uniaxial deformation are drastically different, as shown in Fig. 2(b). In the early stages of post-yield deformation, clear strain hardening is observed for most curves, which is manifested through the nonlinearity of the stress vs strain relationship. Interestingly, larger pillars exhibit a smoother and much more continuous deformation compared with smaller pillars, which show several discrete bursts throughout the process.

The scaling relationship between attained stresses at a particular strain and diameter for both types of crystals clearly shows a power-law-type dependence, as can be seen in the log-log plot in Fig. 3. Because of possible slight misalignment in the initial stages of deformation, we compare flow stresses at $8 \%-10 \%$ strain, when full contact is established, rather than yield stresses, which cannot be unambiguously defined in these experiments. While both crystals exhibit a power-law trend, the scaling slopes are vastly different: -0.97 for gold and -0.47 for molybde-
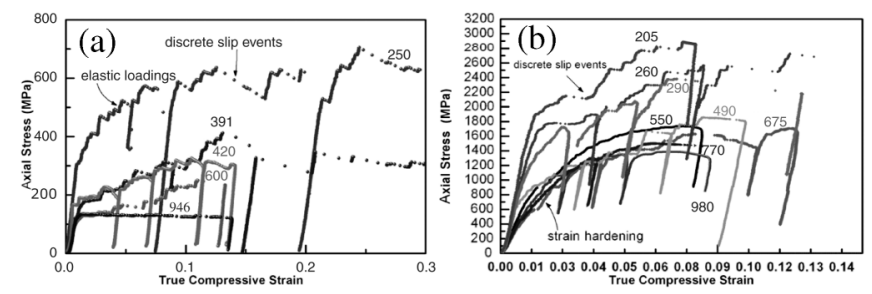

FIG. 2. Some representative stress vs strain curves for (a) fcc $\mathrm{Au}$ and (b) bcc Mo nanopillars. Some Mo and all Au pillars were intentionally unloaded and reloaded several times throughout the experiment. Numbers adjacent to each curve represent the initial diameter of the pillar. num, indicating that the size effect is much more pronounced in fcc crystals compared with bcc. In addition, the smallest $(205 \mathrm{~nm})$ Mo nanopillar reaches the stress of $2.85 \mathrm{GPa}$ compared with $800 \mathrm{MPa}$ for $250 \mathrm{~nm} \mathrm{Au}$ at equivalent strain. These nanopillars can sustain strengths much higher than their bulk equivalents of $\sim 450 \mathrm{MPa}$ for Mo [12] and $25 \mathrm{MPa}$ for Au [13]. With no thermal activation, the absolute flow stress for bulk bcc metals is expected to be much higher than that for fcc metals because of their higher lattice resistance to dislocation motion, i.e., Peierls stress. Since all experiments were performed at room temperature, thermal activation has not rendered the Peierls barrier in Mo ineffective, and its effect is clearly pronounced. In order to estimate the dislocation density evolution in both crystals, it is useful to compare the attained flow stresses to the ideal shear strength, or the stress required to shear one plane of atoms past another in a perfect crystal lattice. Density functional theory predicts that ideal shear strength of Mo on its slip plane is $15.8 \mathrm{GPa}$ and that for $\mathrm{Au}$ is $850 \mathrm{MPa}$ [14], which translates into $37.5 \mathrm{GPa}$ and $1.8 \mathrm{GPa}$ ideal axial strengths for Mo and $\mathrm{Au}$, respectively. The axial stress was calculated from the ideal shear strength by dividing the latter by the Schmid factor for the slip systems considered in the ideal shear strength calculation: $\{1 \overline{1} 0\} /\langle 111\rangle$ for bcc and $\{111\} /\langle 11 \overline{2}\rangle$ for fcc. These findings suggest that fundamentally different dislocation motion mechanisms might be operating in fcc and bcc crystals at nanoscale. The experimental results reported here are consistent with recent computational findings by molecular dynamics and dislocation dynamics simulations [2,8,15-21]. Most of these computational works have primarily addressed the deformation of fcc crystals, where dislocations easily split into ribbons separated by stacking faults, preventing them from cross slipping and restricting them to gliding only in $\{111\}$-type planes [22]. Therefore, in a nanoscale fcc pillar the proximity of any given dislocation to the free surface increases the driving force for it to escape the crystal due to the image forces exerted by the surface. The pillar therefore

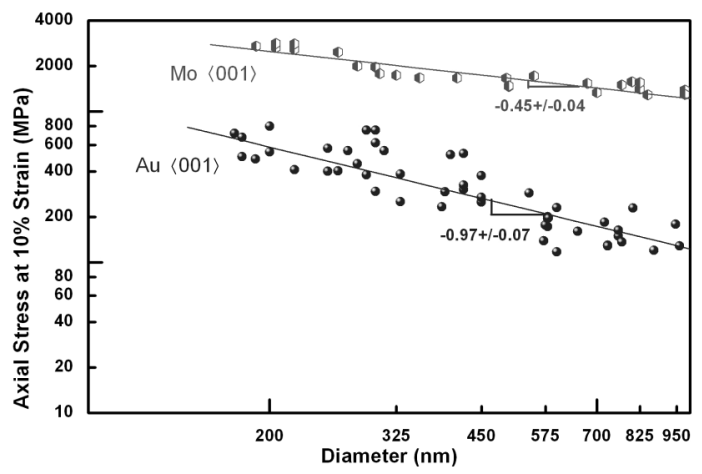

FIG. 3. The log-log plot of flow stress as a function of initial diameter representing the scaling laws for Mo and $\mathrm{Au}$. The slope of strengthening in gold is nearly $2 \times$ higher than that for molybdenum. 
becomes dislocation starved, and new dislocations have to be nucleated to accommodate further plastic deformation [23]. Based on our experimental findings and on the computational predictions, the size effect in fcc materials can be interpreted through dislocation-source limited plasticity unique to nanoscale crystals. To the contrary, in bcc metals, the screw components of a dislocation loop are not restricted to any single glide plane and can therefore move in all 3 dimensions while the edge counterparts tend to be restricted to their glide planes [24-26]. Moreover, the mobility of the screw dislocation segment is significantly slower than that of the edge, thereby increasing the residence time of a dislocation loop inside the pillar [24]. Dislocation dynamics simulations of a 3D cylinder reveal that a single dislocation loop can generate multiple new dislocations and their entanglements, due to the propensity of screw dislocation components to cross slip between different slip planes, as shown in the work of Greer, Weinberger, and Cai [27]. Therefore, despite very low initial dislocation densities present in both metals, their evolution paths are significantly different from each other as well as from bulk. In gold nanopillars, mobile dislocations annihilate at free surfaces upon application of stress, causing the overall dislocation density to decrease, and the associated increase in applied stress is further controlled by nucleation of new dislocation sources most likely at the surface where crystallographic slip steps serve as nucleation sites. In molybdenum, even a single dislocation can replicate itself and leave behind debris in response to the applied stress. The dislocation segments will further interact and form Frank-Read sources, resulting in the everincreasing dislocation density and applied stress. Entanglement of these dislocation segments inside the pillar can contribute to increased flow stress, similar to the forest-hardening model for plasticity of bulk crystals [28].

The burst-ridden characteristic of crystal deformation at nanoscale may represent a fundamental instability in plasticity when nanoscale devices are fabricated. To analyze the discrete nature of stress-strain curves, we employ a two-point forward-Euler time differentiation of the displacement signal. Following the approach of Dimiduk et al. [29] and Csikor et al. [30], the displacement rate shows distinct displacement bursts intermitted by periods of continuous deformation. During the zero-applieddeformation segment after $90 \%$ of load removal, the maximal experimental scatter in the displacement rate was $7.7 \mathrm{~nm} / \mathrm{s}$ and $6.5 \mathrm{~nm} / \mathrm{s}$ for $\mathrm{Au}$ and Mo nanopillars, respectively. Therefore, a threshold of $10 \mathrm{~nm} / \mathrm{s}$ was chosen for both materials to identify true slip events. Integrating the regions above the threshold, the bursts' magnitude is obtained, and the bursts are subsequently binned and normalized according to their size. The distribution of slip event probability as a function of displacement bursts normalized by the Burgers vector $b$ is shown in Fig. 4 .

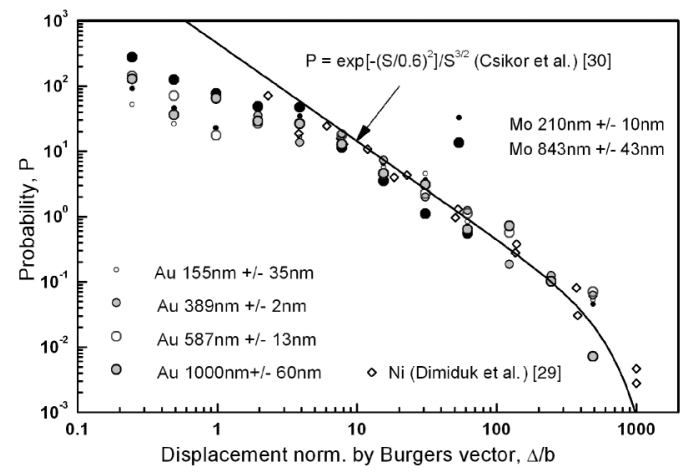

FIG. 4. Statistical distribution of probability of a slip event of a given size vs displacement (normalized by the Burgers vector). Experimental results from this papers are compared to those previously reported as shown on the graph.

For bursts larger than $\sim 10$ Burgers vectors we observe a remarkable agreement with previous reports: the probability distribution is independent of pillar dimensions and of specific material. Moreover, the distribution can be described by a universal scaling function derived based on 3D discrete dislocation dynamics simulations, $P=$ $\exp \left[-\left(S / 0.6^{2}\right)\right] / S^{3 / 2}$, where $S$ represents a characteristic burst size [30]. For these larger displacements, such probability distribution agrees with the notion that occurrence of avalanchelike bursts depends only on the basic plasticity features and is independent of the material. However, our data indicate that, based on this scaling, the probability of avalanchelike plasticity is overestimated when the burst size is below $\sim 10 \mathrm{~b}$. This means that while sample size does not appear to be a key factor in the avalanche probability distribution, slip events of $10 b$ and less are likely to occur in nanosized crystals, and the probability of their occurrence is lower than that predicted by the scaling law. Zaiser et al. [31] found a similar deviation from the scaling law for small slip events in Mo micropillars, with the threshold for slip event identification of $2.5 \mathrm{~nm} / \mathrm{s}$. For smaller thresholds they find that the experimental results agree with the scaling law. Since the choice of threshold might have a significant effect on the validity of the obtained distribution, the threshold value in our study was determined based on setting it higher than the displacement rate noise but without loss of any event larger than that. In addition, this study finds a cutoff for large slip events similar to the predictions of the statistical model $[30,32]$, which was not observed in [31].

In summary, our analysis sheds light onto some of the intricacies of plasticity in nanosized crystals. While many aspects of it are yet to be well understood, our experiments show that both $\mathrm{Au}$ (fcc) and Mo (bcc) exhibit a strong size effect when reduced to nanoscale. Their deformation is controlled by specific dislocation motion characteristic for each crystal, which explains the lack of strain hardening in gold nanopillars and reaching $44 \%$ of its theoretical strength while showing clear strain hardening in molybde- 
num nanopillars, reaching only $\sim 7 \%$ of ideal strength. Avalanchelike displacement burst behavior prevalent in all deformation curves is found to be independent of crystal structure and of specimen size; however, the probability of slip events smaller than $\sim 10$ Burgers vectors is found to be lower than previously predicted.

The authors gratefully acknowledge helpful discussions with C. Weinberger and W. Cai. J.R. G. acknowledges Caltech startup funds.

[1] A. A. Benzerga and N. F. Shaver, Scr. Mater. 54, 1937 (2006).

[2] V. S. Deshpande, A. Needleman, and E. Van der Giessen, J. Mech. Phys. Solids 53, 2661 (2005).

[3] H. D. Espinosa, B. C. Prorok, and B. Peng, J. Mech. Phys. Solids 52, 667 (2004).

[4] K. Gall, J. Diao, and M. Dunn, Nano Lett. 4, 2431 (2004).

[5] J. R. Greer, W. C. Oliver, and W. D. Nix, Acta Mater. 53, 1821 (2005).

[6] M. F. Horstemeyer, M. I. Baskes, and S. J. Plimpton, Acta Mater. 49, 4363 (2001).

[7] M. N. Nadgorny, D. M. Dimiduk, and M. D. Uchic, Mater. Res. Soc. Symp. Proc. 976, EE06 (2007).

[8] L. Nicola, E. Van der Giessen, and A. Needleman, J. Appl. Phys. 93, 5920 (2003).

[9] W. D. Nix, J. R. Greer, G. Feng, and E. Lilleodden, Thin Solid Films 515, 3152 (2007).

[10] H. Tang, K. W. Schwartz, and H. D. Espinosa, Acta Mater. 55, 1607 (2007).

[11] M.D. Uchic, D. M. Dimiduk, J. N. Florando, and W. D. Nix, Science 305, 986 (2004).

[12] F. Guiu and P. L. Pratt, Phys. Status Solidi 15, 539 (1966).

[13] E.M. Savitskii, Handbook of Precious Metals (Hemisphere Publishing Company, New York, 1969), p. 128.
[14] S. Ogata, J. Li, N. Hirosaki, Y. Shibutani, and S. Yip, Phys. Rev. B 70, 104104 (2004).

[15] D. S. Balint, V.S. Deshpande, A. Needleman, and E. Van der Giessen, Model. Simul. Mater. Sci. Eng. 14, 409 (2006).

[16] J. Chang, W. Cai, V. V. Bulatov, and S. Yip, Mater. Sci. Eng. A 309-310, 160 (2001).

[17] B. Devincre, L. P. Kubin, C. Lemarchand, and R. Madec, Mater. Sci. Eng. A 309-310, 211 (2001).

[18] H.D. Espinosa, M. Panico, S. Berbenni, and K.W. Schwartz, Int. J. Plast. 22, 2091 (2006).

[19] P. J. Guruprasad and A. A. Benzerga, J. Mech. Phys. Solids (to be published).

[20] M. Popova, Y.-L. Shen, and T. A. Khraishi, Mol. Simul. 31, 1043 (2005).

[21] E. Rabkin, H. S. Nam, and D. J. Srolovitz, Acta Mater. 55, 2085 (2007).

[22] M.S. Duesbery, Model. Simul. Mater. Sci. Eng. 6, 35 (1998).

[23] J. R. Greer and W. D. Nix, Phys. Rev. B 73, 245410 (2006).

[24] W. Cai and V. V. Bulatov, Mater. Sci. Eng. A 387-89, 277 (2004).

[25] J. Marian, W. Cai, and V. Bulatov, Nat. Mater. 3, 158 (2004).

[26] M. Tang, W. Cai, G. Xu, and V. Bulatov, Model. Simul. Mater. Sci. Eng. 14, 1139 (2006).

[27] J. R. Greer, C. Weinberger, and W. Cai, Mater. Sci. Eng. A (in press).

[28] A. M. Cuitino and M. Ortiz, J. Mech. Phys. Solids 44, 863 (1996).

[29] D. M. Dimiduk, C. Woodward, R. LeSar, and M. D. Uchic, Science 312, 1188 (2006).

[30] F. Csikor, C. Motz, D. Weygand, M. Zaiser, and S. Zapperi, Science 318, 251 (2007).

[31] M. Zaiser, J. Schwerdtfeger, A. S. Schneider, C. P. Frick, B. G. Clark, P. A. Gruber, and E. Arzt (to be published).

[32] M. Zaiser and N. Nikitas, J. Stat. Mech. (2007) P04013. 